\title{
Extremely short duration interval exercise improves 24-h glycaemia in men with type 2 diabetes
}

\author{
Richard S. Metcalfe ${ }^{1}\left(\mathbb{D} \cdot\right.$ Ben Fitzpatrick ${ }^{2} \cdot$ Sinead Fitzpatrick $^{3} \cdot$ Gary McDermott $^{4} \cdot$ Noel Brick $^{5} \cdot$ Conor McClean $^{2}$. \\ Gareth W. Davison ${ }^{2}$
}

Received: 23 May 2018 / Accepted: 25 August 2018 / Published online: 31 August 2018

(c) The Author(s) 2018

\begin{abstract}
Purpose Reduced-exertion high-intensity interval training (REHIT) is a genuinely time-efficient exercise intervention that improves aerobic capacity and blood pressure in men with type 2 diabetes. However, the acute effects of REHIT on 24-h glycaemia have not been examined.

Methods 11 men with type 2 diabetes (mean \pm SD: age, $52 \pm 6$ years; BMI, $29.7 \pm 3.1 \mathrm{~kg} / \mathrm{m}^{2} ; \mathrm{HbA}_{1 \mathrm{c}}, 7.0 \pm 0.8 \%$ ) participated in a randomised, four-trial crossover study, with continual interstitial glucose measurements captured during a 24-h dietarystandardised period following either (1) no exercise (CON); (2) $30 \mathrm{~min}$ of continuous exercise (MICT); (3) $10 \times 1 \mathrm{~min}$ at $~ 90$ $\mathrm{HR}_{\max }$ (HIIT; time commitment, $25 \mathrm{~min}$ ); and (4) $2 \times 20 \mathrm{~s}$ 'all-out' sprints (REHIT; time commitment, $10 \mathrm{~min}$ ).

Results Compared to CON, mean 24-h glucose was lower following REHIT (mean $\pm 95 \% \mathrm{CI}:-0.58 \pm 0.41 \mathrm{mmol} / \mathrm{L}, p=0.008$, $d=0.55)$ and tended to be lower with MICT $(-0.37 \pm 0.41 \mathrm{mmol} / \mathrm{L}, p=0.08, d=0.35)$, but was not significantly altered following HIIT $(-0.37 \pm 0.59 \mathrm{mmol} / \mathrm{L}, p=0.31, d=0.35)$. This seemed to be largely driven by a lower glycaemic response (area under the curve) to dinner following both REHIT and MICT ( $-11 \%, p<0.05$ and $d>0.9$ for both) but not HIIT ( $-4 \%$, $p=0.22, d=0.38$ ). Time in hyperglycaemia appeared to be reduced with all three exercise conditions compared with CON (REHIT: $-112 \pm 63 \mathrm{~min}, p=0.002, d=0.50$; MICT: $-115 \pm 127 \mathrm{~min}, p=0.08, d=0.50$; HIIT $-125 \pm 122 \mathrm{~min}, p=0.04$, $d=0.54$ ), whilst indices of glycaemic variability were not significantly altered.

Conclusion REHIT may offer a genuinely time-efficient exercise option for improving 24-h glycaemia in men with type 2 diabetes and warrants further study.
\end{abstract}

Keywords Exercise $\cdot$ High-intensity interval training $\cdot$ Postprandial glucose $\cdot$ Continuous glucose monitoring $\cdot$ Type 2 diabetes

Communicated by Phillip D Chilibeck.

Richard S. Metcalfe

r.s.metcalfe@swansea.ac.uk

1 Applied Sports Technology, Exercise and Medicine Research Centre (A-STEM), Swansea University, Bay Campus, Fabian Way, Swansea SA1 8EN, UK

2 Sport and Exercise Science Research Institute, Ulster University, Belfast, Northern Ireland, UK

3 Western Health and Social Care Trust, Altnagelvin Area Hospital, Derry, Northern Ireland, UK

4 School of Sport, Ulster University, Belfast, Northern Ireland, UK

5 Psychology Research Institute, Ulster University, Belfast, Northern Ireland, UK

$\begin{array}{ll}\text { Abbreviations } \\ \text { ADA } & \text { American Diabetes Association } \\ \text { AUC } & \text { Area under the curve } \\ \text { iAUC } & \text { Incremental area under the curve } \\ \text { BMI } & \text { Body mass index } \\ \text { CGM } & \text { Continuous glucose monitoring } \\ \text { CONGA } & \text { Continuous net overlapping glucose action } \\ \text { HbA }_{1 c} & \text { Glycated hemoglobin } \\ \text { HIIT } & \text { High-intensity interval training } \\ \text { HR } & \text { Heart rate } \\ \text { HRmax } & \text { Maximal heart rate } \\ \text { MAGE } & \text { Mean amplitude of glucose excursions } \\ \text { MICT } & \text { Moderate-intensity continuous training } \\ \text { PA } & \text { Physical activity } \\ \text { PAL } & \text { Physical activity level } \\ \text { REHIT } & \text { Reduced-exertion high-intensity interval } \\ & \text { training }\end{array}$


SIT Sprint interval training

$\mathrm{VO}_{2} \quad$ Oxygen uptake

$V \mathrm{O}_{2 \text { peak }} \quad$ Peak oxygen uptake

$W_{\max } \quad$ Peak power output

\section{Introduction}

Exaggerated postprandial glycaemic excursions are strongly correlated with type 2 diabetes-related complications, including cardiovascular disease, which is a major cause of morbidity and mortality (Monnier and Colette 2015). However, studies employing continuous glucose monitoring (CGM) have shown that, despite pharmacological intervention, a large proportion of patients with type 2 diabetes (and even those well controlled according to $\mathrm{HbA}_{1 \mathrm{c}}$ ) still spend significant portions of the day in hyperglycaemia (van Dijk et al. 2011). This emphasises the importance of a multi-component treatment approach in type 2 diabetes, incorporating regular exercise, which is an effective strategy for lowering postprandial glucose excursions (Van Dijk et al. 2013) and $\mathrm{HbA}_{1 \mathrm{c}}$ (Church et al. 2010) over and above improvements seen with first-line drug therapies. Whilst exercise traininginduced adaptations may result in an additive and more prolonged improvement in insulin sensitivity (i.e. $>72 \mathrm{~h}$ posttraining) (Dela et al. 1995), it is generally accepted that the cumulative impact of regular (i.e. daily) acute exercise on glycaemic control (van Dijk et al. 2012) is of greater clinical importance for the long-term management of glycaemic control in type 2 diabetes (Colberg et al. 2016).

The exercise recommendations for individuals with type 2 diabetes are similar as for the general population, suggesting a minimum of 150 min of moderate-vigorous-intensity aerobic exercise each week (Colberg et al. 2016; Garber et al. 2011). However, self-report data suggest that adherence to these guidelines is poor in the general population (Allender et al. 2008; Hallal et al. 2012) and potentially even lower in those with type 2 diabetes (Morrato et al. 2007). The reasons for poor exercise adherence are numerous and complex, but a perceived lack of time is consistently reported as one of the important barriers in people with type 2 diabetes (Korkiakangas et al. 2009). In response to this, submaximal highintensity interval training (HIIT) and supramaximal sprint interval training (SIT) have been proposed as time-efficient alternative exercise options for improving glycaemic control. Acute studies in overweight individuals (Little et al. 2014) and people with type 2 diabetes (Terada et al. 2016) have shown superior improvements in glycaemic control with HIIT compared with 30-60 min of traditional MICT. Despite the case for a superior clinical benefit, the total time commitment, including recovery intervals, means that most HIIT protocols are not as time-efficient as often claimed. To date, the protocols studied generally require $20-60 \mathrm{~min}$
(Terada et al. 2016; Little et al. 2011; Gillen et al. 2012), which is no different (and in some cases, exceeds) than current exercise recommendations for MICT (Colberg et al. 2016; Garber et al. 2011). Moreover, there is currently vigorous debate about whether either HIIT or SIT would be appropriate exercise strategies for recommendation to the general population or specific patient populations, based on the hypothesised potential for 'unpleasant' perceptual responses (e.g. high perceived exertion and negative affect) to lead to low exercise adherence (Hardcastle et al. 2014). The total time commitment and potential for unpleasant perceptual responses increase as a function of the number and duration of high-intensity efforts (Townsend et al. 2017). Thus, it is important to examine whether HIIT/SIT protocols, with fewer and shorter high-intensity efforts, remain efficacious for improving insulin sensitivity and glycaemic control in type 2 diabetes (Vollaard and Metcalfe 2017).

There is evidence that HIIT/SIT protocols with fewer and/ or shorter sprints can improve glucose tolerance in healthy sedentary individuals (Metcalfe et al. 2012, 2016; Gillen et al. 2016). For example, we previously demonstrated that a modified SIT intervention, consisting of $10 \mathrm{~min}$ of lowintensity cycling interspersed with two $20 \mathrm{~s}$ 'all-out' sprints [termed 'reduced-exertion high-intensity interval training' (REHIT)], was effective at improving insulin sensitivity in sedentary men over 6 weeks (Metcalfe et al. 2012). Importantly, these benefits were observed despite the low time commitment (10 min per session) and acceptable session ratings of perceived exertion ('somewhat hard'). Ruffino et al. (2017) recently applied REHIT in type 2 diabetes and observed superior improvements in aerobic capacity and similar changes in resting blood pressure compared with a moderate-intensity walking intervention over an 8-week training period. REHIT did not improve insulin sensitivity or reduce 24-h mean glucose in this study, however, responses were captured 3 days following training cessation and improvements may have been lost at this time point (Ruffino et al. 2017). Nevertheless, 8 weeks of REHIT did lower plasma frucotosamine concentrations (a marker of average blood glucose levels in the preceding 2-4-week period), suggesting improved glycaemic control during the training intervention (Ruffino et al. 2017). Yet, the acute effects of REHIT on post-exercise glycaemic control in people with type 2 diabetes have not been explored. Thus, the primary aim of this study was to examine the effect of a single bout of REHIT on 24-h glycaemia in men with type 2 diabetes relative to a no-exercise control trial using continuous glucose monitors. Our primary hypothesis was that REHIT would improve glycaemic control relative to no exercise. We also studied the effects of a single bout of MICT and a single bout of HIIT compared with no exercise, as both have previously been shown to improve glycaemic control (Gillen et al. 2012; van Dijk et al. 2012). 


\section{Methods}

\section{Ethical approval}

This randomised-controlled acute crossover trial was conducted at Ulster University, Northern Ireland (UK), between October 2016 and August 2017 (ClinicalTrials.gov registration: NCT03082859). The experimental protocol was approved by the Office for Research Ethics in Northern Ireland (RECA ref: 16/NI/0115) and conducted in accordance with the Declaration of Helsinki.

\section{Participants}

$11(n=11)$ men, diagnosed with type 2 diabetes mellitus by a clinician $4 \pm 3$ (range $0.5-8$ ) years previously, completed the full experimental procedures (Fig. 1; Table 1). Exclusion criteria included exogenous insulin therapy, currently taking more than two glucose-lowering medications, BMI $\geq 40 \mathrm{~kg} /$ $\mathrm{m}^{2}$, classification as highly active on the international physical activity questionnaire (IPAQ) (Craig et al. 2003), and any contraindications to exercise, including any history of cardiovascular or cerebrovascular disease, impaired renal or liver function, and hypertension not well controlled by standard medication. All participants were informed about the study, both verbally and in writing, before providing their written consent to participate. Eligible participants completed a 12-lead exercise stress test on a cycle ergometer (Lode Corival; Lode, Groningen, Netherlands) and received clearance for vigorous-intensity exercise from a clinical cardiac physiologist. At baseline, all medication was recorded and
Table 1 Participant characteristics $(n=11)$

\begin{tabular}{ll}
\hline Characteristics & Mean \pm SD (range) \\
\hline Age (years) & $52 \pm 6(40-60)$ \\
Body mass $(\mathrm{kg})$ & $91.8 \pm 10.4(65.6-100.9)$ \\
Height $(\mathrm{m})$ & $1.76 \pm 0.07(1.66-1.89)$ \\
$\mathrm{BMI}\left(\mathrm{kg} / \mathrm{m}^{2}\right)$ & $29.7 \pm 3.1(23.8-34.1)$ \\
$\mathrm{VO}_{2 \mathrm{max}}(\mathrm{ml} / \mathrm{kg} / \mathrm{min})$ & $28.9 \pm 4.8(18.3-35.3)$ \\
Systolic blood pressure $(\mathrm{mmHg})$ & $125 \pm 12(114-148)$ \\
Diastolic blood pressure $(\mathrm{mmHg})$ & $78 \pm 7(70-88)$ \\
Mean arterial pressure & $93 \pm 8(83-108)$ \\
Duration of T2D (years) & $4 \pm 3(0.5-8)$ \\
Fasting glucose (mmol/l) & $8.1 \pm 1.2(7.1-9.7)$ \\
HbA $\mathrm{A}_{\mathrm{lc}}(\mathrm{mmol} / \mathrm{mol})$ & $52 \pm 9(38-67)$ \\
HbA $1 \mathrm{cc}(\%)$ & $7.0 \pm 0.8(5.6-8.3)$ \\
Medication $(n)$ & \\
Metformin & 7 \\
Metformin + sulfonylurea & 4 \\
Blood pressure lowering & 1 \\
Statin & 5 \\
\hline
\end{tabular}

$T 2 D$ type 2 diabetes

participants were instructed to maintain their usual dose/ timing/type of medication throughout the study period.

\section{Pre-experimental procedures}

Following health screening, participants completed a maximal incremental cycling test to volitional exhaustion to determine peak oxygen uptake $\left(V \mathrm{O}_{2 \text { peak }}\right)$, peak power output $\left(W_{\max }\right)$ and peak heart rate $\left(\mathrm{HR}_{\max }\right)$. Following a 5-min

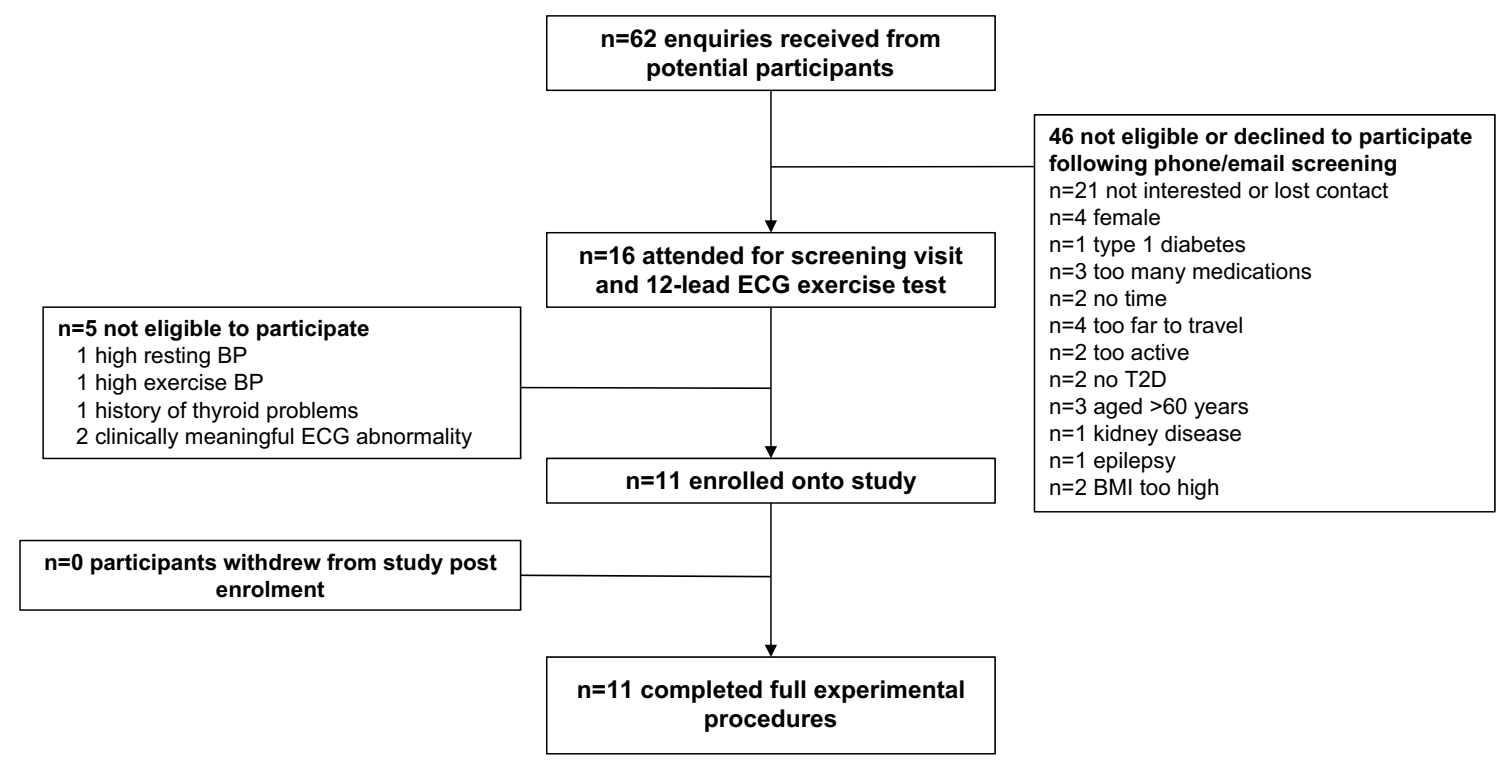

Fig. 1 Flow of participants through the study 
warm up at $50 \mathrm{~W}$, the intensity was increased by $15 \mathrm{~W} / \mathrm{min}$ until cadence could not be maintained at $\geq 50 \mathrm{rpm}$ (Lode Corival). $V \mathrm{O}_{2 \text { peak }}$ was determined as the highest ten-breath rolling average, $V \mathrm{O}_{2}$ measured using an online gas analysis system (Cosmed Quark; CPET, Rome, Italy), and accepted if two or more of the following criteria were met: (1) volitional exhaustion, (2) a plateau in $V \mathrm{O}_{2}$ despite increasing intensity, (3) RER $>1.15$, and (4) maximal heart rate within 10 beats of the age-predicted maximum (i.e. 220-age) (Howley et al. 1995). This was the case for all participants. Participants also completed two familiarisation sessions, on separate days, each lasting approximately $20 \mathrm{~min}$, to introduce participants to the technique and effort required to perform allout cycling sprints.

\section{Main experimental protocol}

Each participant completed four main experimental trials (CON, REHIT, HIIT and MICT) in a randomised order (envelope method), with each trial taking place over 3 days. During each trial, participants underwent $24 \mathrm{~h}$ of continuous glucose monitoring under standardised dietary intake and the following experimental conditions: (1) a no-exercise control condition (CON); (2) a single bout of high-intensity interval training (HIIT); (3) a single bout of reduced-exertion high-intensity interval training (REHIT); and (4) moderate-vigorous-intensity continuous exercise (MICT). Each trial was separated by at least 5 days, and prior to each trial, participants were asked to avoid any structured exercise for 2 days. This was confirmed via physical activity monitoring using synchronised accelerometry and heart rate monitoring with branched model equations (Actiheart, Cambridge Neurotechnology Ltd., Cambridge, UK). The Actiheart is a non-invasive physical activity monitor that is both reliable and valid, can accurately estimate energy expenditure across low-, moderate- and high-intensity physical activities, and provides useful quantitative data on patterns of physical activity, allowing a comprehensive characterisation of physical activity status (Thompson et al. 2006). Participants wore the monitor continuously (day and night) and were instructed to only remove it when showering or bathing. There were no differences in physical activity across the 2 days prior to each experimental trial $(p>0.05$ for all main effects of condition, respectively; Table 2). The Actiheart monitors were also worn throughout each main 24-h monitoring period.

Participants attended the lab between 15:00 and 18:00 $\mathrm{h}$ on day 1 for insertion of a subcutaneous glucose sensor (Enlite, Medtronic Inc., Minneapolis, USA) and CGM in the abdomen (iPro2, Medtronic Inc. Minneapolis, USA). Sensors were inserted approximately $5 \mathrm{~cm}$ from the umbilicus and on the opposite side from which participants tended to sleep on. The iPro2 measures glucose in the interstitial fluid
Table 2 Dietary composition for the pre-trial evening meal and the main trial day

\begin{tabular}{llc}
\hline & $\begin{array}{l}\text { Pre-trial even- } \\
\text { ing meal (day } \\
1 \text { ) }\end{array}$ & Main trial day (day 2) \\
\hline Total energy content & $796 \pm 187$ & $2441 \pm 235$ \\
CHO (kcal) & $365 \pm 79$ & $1243 \pm 100$ \\
Fat (kcal) & $261 \pm 99$ & $761 \pm 156$ \\
Pro (kcal) & $171 \pm 49$ & $436 \pm 49$ \\
CHO (\%) & $47 \pm 8$ & $51 \pm 3$ \\
Fat (\%) & $32 \pm 7$ & $31 \pm 4$ \\
Pro (\%) & $21 \pm 4$ & $18 \pm 2$ \\
Breakfast (\% of total energy) & - & $20 \pm 5$ \\
Lunch (\% of total energy) & - & $29 \pm 4$ \\
Dinner (\% of total energy) & - & $30 \pm 5$ \\
Snacks (\% of total energy) & - & $21 \pm 6$ \\
\hline
\end{tabular}

Data shown as mean $\pm \mathrm{SD}$

$\mathrm{CHO}$ carbohydrate, Pro protein

every $5 \mathrm{~min}$ and values are subsequently converted to blood glucose using capillary measurements taken by each participant before each main meal and before sleep (Accu-Check; Roche Diagnostics, Basel, Switzerland). It has previously shown good validity and reliability when compared to blood glucose measured simultaneously from an intravenous cannula (Bailey et al. 2014). Participants then returned home but were provided with a standardised evening meal and snack (Table 2).

Participants returned to the laboratory the following morning (day 2) between 07:00 and 08:30 $\mathrm{h}$ to consume breakfast and complete the exercise session (30 min postbreakfast). During CON, participants remained sedentary throughout this entire period (i.e. $\sim 2$ h). Participants then returned to their normal daily routine but were provided with standardised meals (lunch, evening meal, snacks, to be consumed at standardised times (Table 2)). Participants returned to the laboratory the following morning for removal of the CGM.

\section{Dietary standardisation}

Participants were provided with a standardised food and (energy containing) drink packages containing meals, snacks and drinks for each $42 \mathrm{~h}$ trial period ( 18:00 $\mathrm{h}$ on day 1 to $\sim$ 12:00 $\mathrm{h}$ on day 3 ; Table 3 ). The diet was designed to meet resting metabolic rate [determined using the Harris and Benedict equation (Harris and Benedict 1918)] multiplied by a PA level of 1.4. The macronutrient content was composed according to the 2008 ADA dietary recommendations for type 2 diabetes (Bantle et al. 2008) and consisted of three meals and snacks per full day. The contents of the dietary package were self-selected by participants (in consultation 
Table 3 Actiheart-derived physical activity energy expenditure prior to and during each experimental trial

\begin{tabular}{|c|c|c|c|c|c|c|c|c|}
\hline & \multicolumn{4}{|c|}{ Pre-trial (2 days) } & \multicolumn{4}{|c|}{ Main trial day } \\
\hline & $\mathrm{CON}$ & REHIT & HIIT & MICT & $\mathrm{CON}$ & REHIT & HIIT & MICT \\
\hline TEE (Kcal/day) & $2869 \pm 363$ & $2847 \pm 319$ & $3000 \pm 487$ & $2889 \pm 307$ & $2719 \pm 258$ & $2913 \pm 435$ & $3325 \pm 641^{*}$ & $3225 \pm 356^{*}$ \\
\hline PAL & $1.49 \pm 0.18$ & $1.48 \pm 0.17$ & $1.56 \pm 0.24$ & $1.51 \pm 0.15$ & $1.41 \pm 0.12$ & $1.51 \pm 0.21$ & $1.73 \pm 0.32 *$ & $1.69 \pm 0.16^{*}$ \\
\hline Sedentary time (mins) & $1107 \pm 119$ & $1091 \pm 139$ & $1062 \pm 155$ & $1083 \pm 132$ & $1166 \pm 91$ & $1091 \pm 120$ & $1000 \pm 146^{*}$ & $1006 \pm 95^{*}$ \\
\hline Light PA (mins) & $244 \pm 84$ & $271 \pm 99$ & $265 \pm 108$ & $263 \pm 96$ & $212 \pm 62$ & $257 \pm 72 *$ & $282 \pm 72 *$ & $294 \pm 69 *$ \\
\hline Moderate PA (mins) & $85 \pm 55$ & $77 \pm 53$ & $107 \pm 77$ & $93 \pm 45$ & $61 \pm 43$ & $87 \pm 65$ & $135 \pm 96^{\wedge}$ & $119 \pm 52^{\wedge}$ \\
\hline Vigorous PA (mins) & $4 \pm 11$ & $1 \pm 1$ & $6 \pm 14$ & $1 \pm 2$ & $1 \pm 1$ & $5 \pm 7$ & $21 \pm 16^{*}$ & $21 \pm 24$ \\
\hline
\end{tabular}

Data shown are mean $\pm \mathrm{SD}$

$T E E$ total energy expenditure, $P A L$ physical activity level, $P A$ physical activity

${ }^{*} p<0.05$ vs control

${ }^{\wedge} p=0.06$ vs control

with the principle investigator) from a pre-determined list of foods available from a local supermarket. In this way, individual food preferences and tolerances were taken into account but the investigators were able to ensure appropriate energy and macronutrient content (Bantle et al. 2008). The food and drinks were ingested at pre-determined times for each participant so that a completely standardised diet was consumed during all four experimental trials. In addition to any energy-containing drinks provided to participants in food packages, participants were allowed to consume water ad libitum throughout each trial.

\section{Exercise bouts}

\section{REHIT}

The REHIT bout was performed on a mechanically braked cycle ergometer (Monark Peak Bike; Monark, Vansbro, Sweden) and was based on the protocol used in previous studies (Metcalfe et al. 2012, 2015, 2016; Ruffino et al. 2017). It consisted of 10 min of unloaded pedalling interspersed with two 'all-out' sprints against a resistance equivalent to $5 \%$ of body mass. Just before each sprint, participants increased their pedal cadence to their maximal speed; the braking force was applied to the ergometer and participants maintained the highest possible cadence against the resistance for $20 \mathrm{~s}$. Sprints were performed at $2 \mathrm{~min} 40 \mathrm{~s}$ and $6 \mathrm{~min} 40 \mathrm{~s}$ into the 10-min exercise session.

\section{HIIT}

The HIIT bout was performed on an electronically braked cycle ergometer (Lode Corival; Lode, Groningen, Netherlands) and involved $10 \times 60 \mathrm{~s}$ cycling efforts interspersed with $60 \mathrm{~s}$ of low-intensity recovery $(25 \mathrm{~W})$ as previously described (Little et al. 2011; Gillen et al. 2012). Individual workloads were set at $85 \% W_{\max }$, as pilot work has suggested this was appropriate for achieving $~ 90 \%$ maximal heart rate $\left(\mathrm{HR}_{\max }\right)$ during the final intervals.

\section{MICT}

MICT was performed on an electronically braked cycle ergometer (Lode Corival; Lode, Groningen, Netherlands) and involved $30 \mathrm{~min}$ of continuous cycling at an intensity equivalent to $50 \%$ of $W_{\max }$ with a 2 -min warm up and cool down (25 W) as previously described (van Dijk et al. 2012).

\section{Calculations}

The 24-h period of interest for continuous glucose measurements commenced at the start of the breakfast period on day 2. The continuous glucose data were exported to Excel (Microsoft, Washington, USA), the relevant 288, 5-min replicate values were isolated, and subsequently converted in summary variables including mean 24-h glucose (primary outcome), and secondary outcomes including glycaemic variability, proportion of time in hyperglycaemia, 24-h incremental (above fasting; calculated from the mean glucose 30 min prior to breakfast) AUC (iAUC), and the total AUC for $3 \mathrm{~h}$ breakfast, lunch and dinner responses. AUC was calculated using the trapezoid rule. The 3 -h postprandial glucose (3-h PPG) was defined as mean glucose 150-180 min following each main meal. The glycaemic range, the mean amplitude of glycaemic excursions (MAGE) and the continuous overall net glycaemic action (CONGA) were calculated as measures of glycaemic variability (Rodbard 2009) using a freely available Excel Macro (Easy GV 9.0; available at http://www.easygv.co.uk). The hyperglycaemic threshold was defined as $\geq 9 \mathrm{mmol} / \mathrm{l}$ based on the published International Diabetes Federation criteria (International Diabetes Federation 2014).

For the analysis of the Actihearts, minute by minute energy expenditure was calculated using the manufacturers 
software (Actiheart, Cambridge Neurotechnology Ltd., Cambridge, UK) and subsequently exported to Microsoft Excel for determination of physical activity summary variables including total 24-h energy expenditure (TEE), physical activity level (PAL; total energy expenditure / resting energy expenditure), sedentary time (mins $<1.5$ METs), time in light PA (mins $>1.5$ METs but $<3$ METs), time in moderate PA (mins $>3$ METs and $<6$ METs) and time in vigorous PA (mins > 6 METs) (Haskell et al. 2007; Pate et al. 2008).

\section{Statistical analysis}

All statistical analysis was performed in GraphPad Prism 7 for Mac OS X. Differences between conditions for exercise responses (i.e. power output and exercise energy expenditure), as well as for 24-h CGM and PA summary variables, were compared using a one factor (condition) repeated measures ANOVA. ANOVA was performed regardless of any minor deviances from a normal distribution and with the Greenhouse-Geisser correction applied in cases of violated sphericity (Maxwell and Delaney 2004). If significant main effects were observed for 24-h CGM and PA variables, then to address our planned primary aim and hypothesis we compared each of the three exercise conditions to control with a paired t-test and a Bonferroni correction for multiple comparisons. For comparisons of exercise responses, the oneway ANOVA was followed up with Bonferroni-corrected $t$ tests to locate differences between exercise conditions. Statistical significance was set at $p \leq 0.05$ (two-tailed) and, unless stated otherwise, data are presented as mean \pm SD. Cohens $d$ was calculated as a measure of effect size with the following thresholds: small $(d=0.2)$, medium $(d=0.6)$ and large $(d=1.2)$ effect.

\section{Results}

\section{Exercise characteristics/intervention fidelity}

All participants successfully completed the three exercise sessions. During the exercise work intervals, mean power output was lowest during MICT $(97 \pm 17 \mathrm{~W})$, higher during HIIT $(165 \pm 28 \mathrm{~W}, p<0.05$ vs MICT), and higher still following REHIT $(417 \pm 49 \mathrm{~W}, p<0.05$ vs HIIT and MICT). On the other hand, Actiheart-estimated energy expenditure during exercise was, on average, lowest during REHIT $(251 \pm 94 \mathrm{~kJ})$, higher during HIIT $(921 \pm 279 \mathrm{~kJ}$, $p<0.05$ vs REHIT), and tended to be highest during MICT $(1076 \pm 378 \mathrm{~kJ}, p<0.05$ vs REHIT, $p=0.07$ vs HIIT). During REHIT, peak, average and end power output were $5.9 \pm 0.7,4.8 \pm 0.6$ and $3.7 \pm 0.6 \mathrm{~W} / \mathrm{kg}$ for the first sprint, and $5.6 \pm 0.5,4.4 \pm 0.5$ and $3.2 \pm 0.6 \mathrm{~W} / \mathrm{kg}$ for the second sprint, respectively.

MICT elicited a mean exercise heart rate of $80 \pm 5 \%$ of the $\mathrm{HR}_{\text {max }}$ achieved during the $V \mathrm{O}_{2 \text { peak }}$ test, whilst during the HIIT work intervals there was a progressive increase in heart rate, which reached $89 \pm 5 \%, 90 \pm 5 \%, 91 \pm 5 \%, 92 \pm 4 \%$ and $94 \pm 5 \%$ of $\mathrm{HR}_{\max }$ during intervals 6-10, respectively (Fig. 2). Heart rate during REHIT peaked at $86 \pm 4 \%$ and $91 \pm 3 \%$ of $\mathrm{HR}_{\max }$ during sprint 1 and 2 , respectively, but mean exercise heart rate during REHIT was $74 \pm 12 \%$ of $\mathrm{HR}_{\max }$ (Fig. 2).

The impact of the four-trial conditions on 24-h physical activity energy expenditure derived from the Actihearts is shown in Table 2. There were significant main effects of condition for all PA parameters ( $p<0.05$ for all). Both HIIT and MICT appeared to increase total 24-h energy expenditure ( $p<0.05$ for both), light PA ( $p<0.05$ for both), moderate PA ( $p=0.06$ for both), and decrease sedentary time $(p<0.05$ for both), when compared with CON. Vigorous PA was significantly increased with HIIT compared with CON only $(p<0.05)$. Although 24 -h TEE, light PA, moderate PA, and vigorous PA were higher, and sedentary time lower, on average with REHIT compared with CON, the differences were smaller and [with the exception of light PA $(p<0.05)$ ] not statistically significant.

\section{Effects of exercise on glycaemic parameters}

\section{4-h summary variables}

The mean effect of exercise on 24-h glycaemic summary variables is shown in Table 4 with individual participant change scores (exercise minus control) for key summary

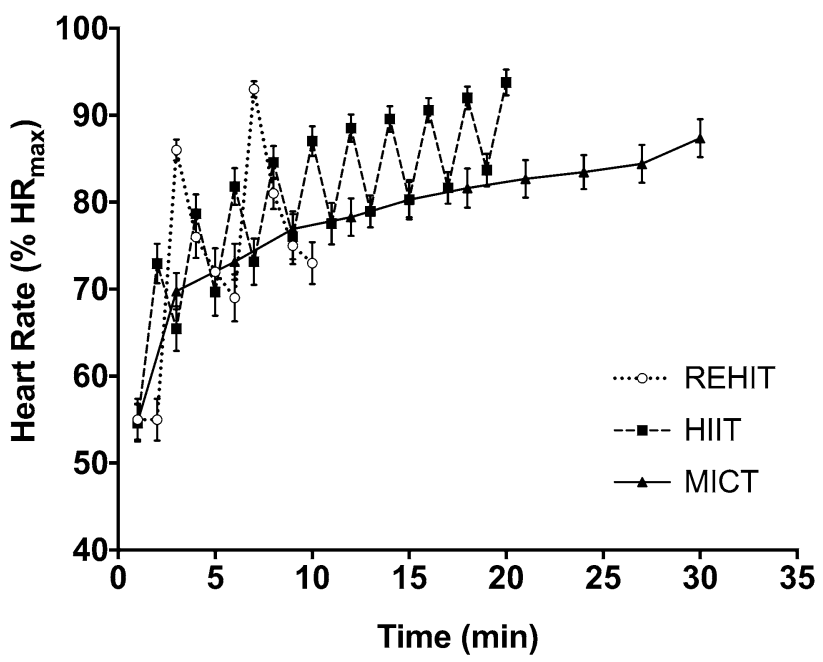

Fig. 2 Heart rate responses over time with each exercise session. Data are presented as mean and SEM for visual clarity 


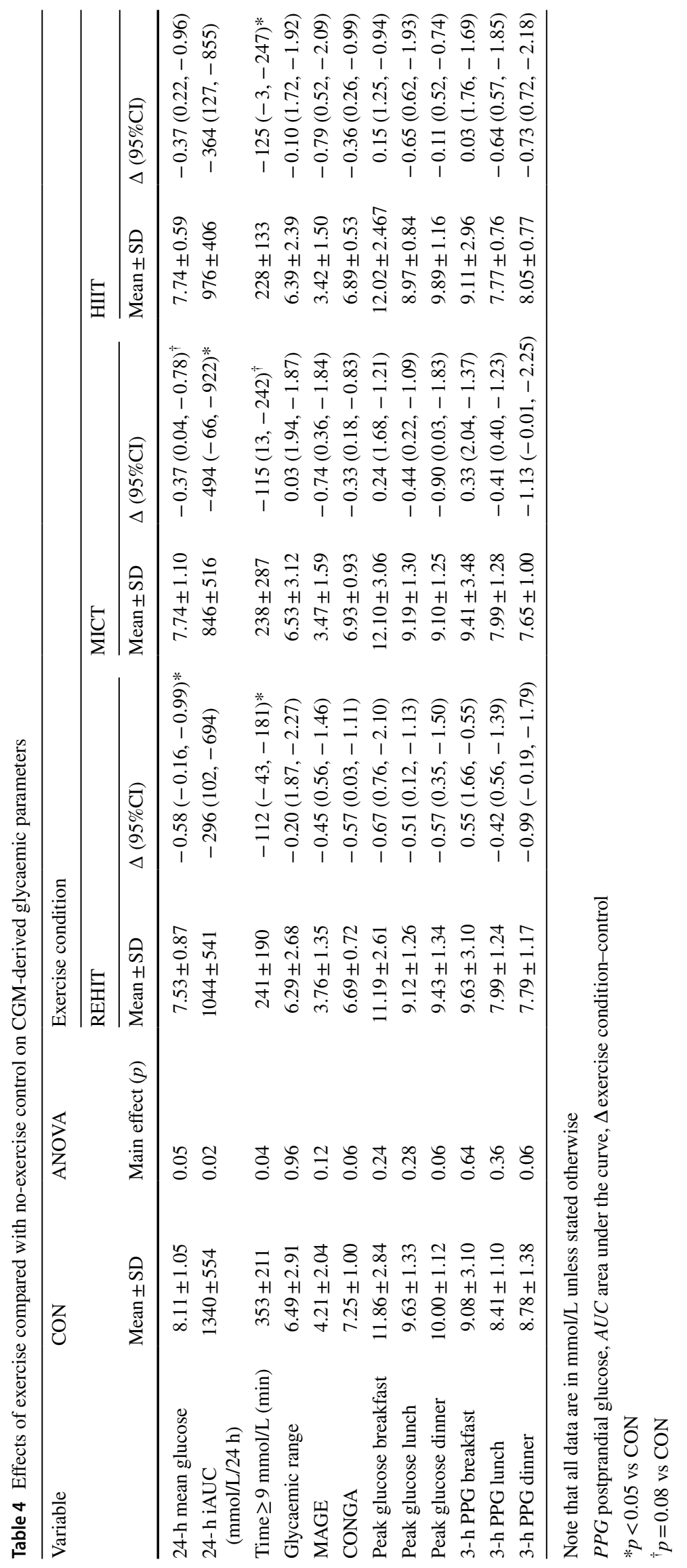



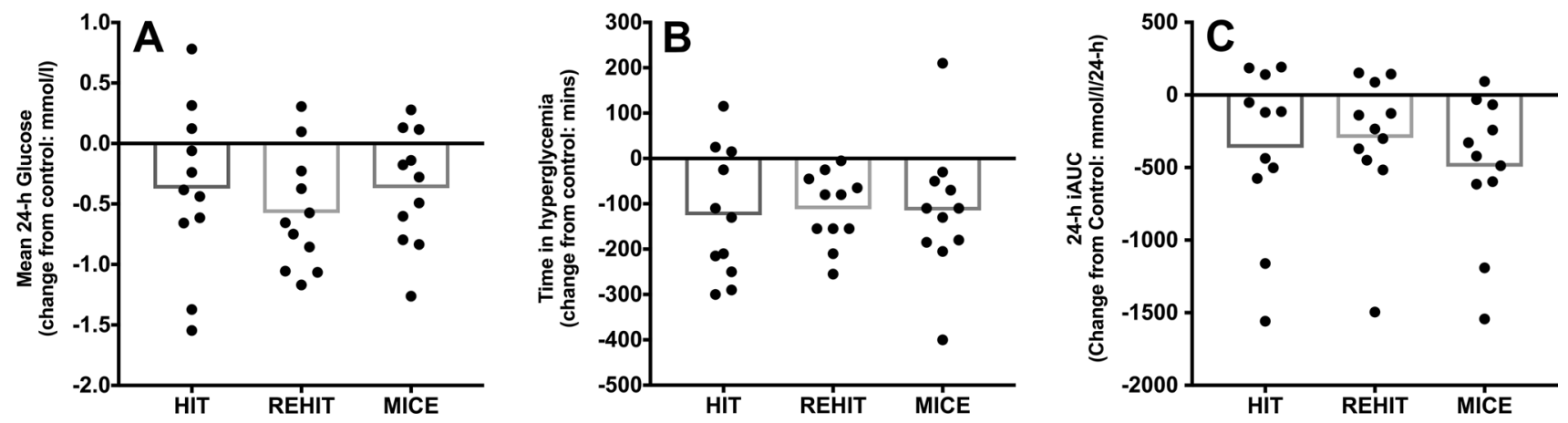

Fig. 3 Effect of exercise on 24-h CGM summary variables. Data are presented as mean change (bars) with individual change scores (dots) compared with CON

variables shown in Fig. 3. There were significant effects of condition for mean 24-h glucose ( $p=0.05)$, time in hyperglycaemia $(p=0.04)$ and for 24-h iAUC $(p=0.02)$. Mean 24-h glucose was lower during REHIT $(p=0.008, d=0.55)$ and tended to be lower during MICT ( $p=0.08, d=0.35$ ) when compared to CON, but there was no statistically significant change observed with HIIT ( $p=0.31, d=0.35$ ). Time spent in hyperglycaemia appeared to be lower following all three exercise conditions compared with CON (REHIT: $p=0.002$, $d=0.50$; MICT: $p=0.08, d=0.50$; HIT: $p=0.04, d=0.54$ ), whilst 24-h iAUC was significantly reduced following MICT only ( $p=0.02, d=0.89)$. There were no significant changes in 24-h SD, glycaemic range, MAGE or CONGA with any of the exercise conditions ( $p>0.05$ for all main effects, respectively; Table 4).

\section{Meal responses}

The 3-h glycaemic responses to breakfast, lunch and dinner are shown in Fig. 4 with additional postprandial summary variables in Table 4 . There were no differences between conditions in the glycaemic response to breakfast or lunch (both $p>0.05$, respectively). However, there was a significant effect of condition on the AUC for dinner $(p=0.004)$, with lower AUC following REHIT $(-11 \%, p=0.018$, $d=1.05)$ and MICT $(-11 \%, p=0.006, d=0.99)$ compared with CON. The glycaemic response to dinner was not significantly affected by HIIT $(-4 \%, p=0.22, d=0.38)$. There were no significant main effects of condition on any other postprandial variable (Table 4).

\section{Adverse events}

One participant reported subjective symptoms of hypoglycaemia between lunch and dinner during the HIIT trial; however, glucose recorded via the CGM appeared to be within the normal range (between 5 and $6 \mathrm{mmol} / \mathrm{l}$ ). There were no other adverse events.

\section{Discussion}

This study examined the acute effects of three discordant exercise strategies, performed after breakfast, on CGMderived 24-h glycaemic control in 11 men with type 2 diabetes. We replicate the findings of numerous previous studies that have shown the beneficial effects of a single 30-min bout of MICT on the 24-h glycaemic profile in type 2 diabetes (van Dijk et al. 2012, 2013), and provide the first evidence to suggest that a modified SIT protocol (REHIT), requiring $40 \mathrm{~s}$ of high-intensity exercise within a total time commitment of $10 \mathrm{~min}$, is also associated with positive glycaemic effects in the post-exercise period. This finding is both novel and of potential significance, as it provides the first evidence for a genuinely time-efficient exercise option to improve glycaemic control in individuals with type 2 diabetes who currently perceive lack of time as a barrier to performing regular structured exercise (Korkiakangas et al. 2009).

REHIT was associated with small (Cohens $d$ between 0.2 and 0.6) but significant beneficial decreases in 24-h mean glucose and the prevalence of hyperglycaemia relative to a no-exercise control trial. This appears to have been predominantly driven by a marked reduction in the glycaemic response to the evening meal, as the breakfast and lunch responses were not significantly affected. Importantly, the improvements in glycaemic control were observed in addition to the impact of participants' current medication, given the response to the no exercise (i.e. medication only) control trial. It is well established that hyperglycaemia is associated with endothelial cell stress and subsequent vascular dysfunction (Paneni et al. 2013), whilst improving glycaemic control reduces the risk of microvascular complications in type 2 diabetes (Stolar 2010). This is largely based on analysis of fasting glucose concentrations, OGTT glucose responses, or $\mathrm{HbA}_{1 c}$, as estimates of glycaemic control, so it is difficult to draw direct comparisons on relative risk reduction for microvascular complications based on CGM variables (Monnier and Colette 2015). For example, a reduction in 

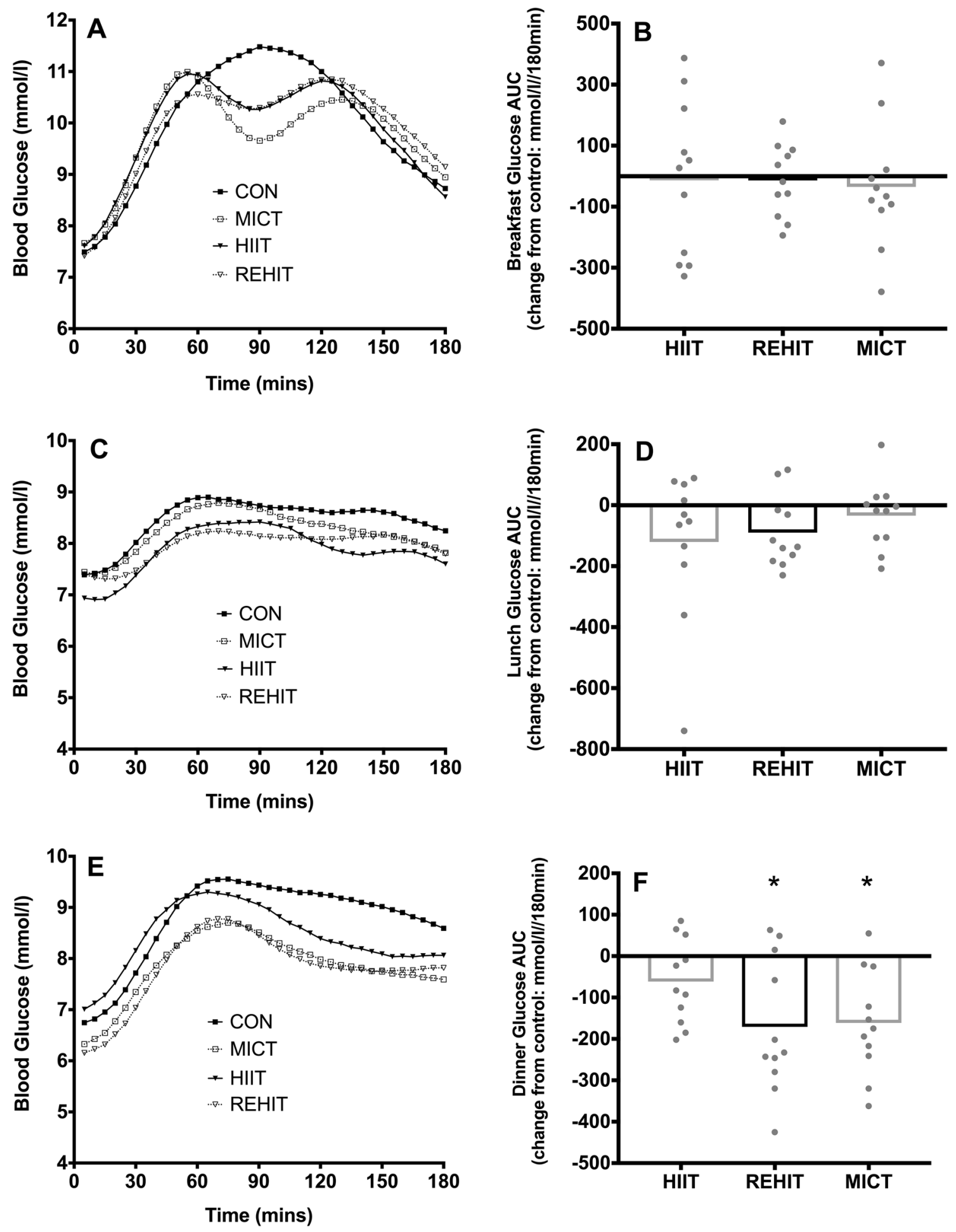

Fig. 4 Glucose time responses (a, c, e) and $\operatorname{AUC}(\mathbf{b}, \mathbf{d}, \mathbf{f})$ for breakfast $(\mathbf{a}, \mathbf{b})$, lunch $(\mathbf{c}, \mathbf{d})$ and dinner $(\mathbf{e}, \mathbf{f})$. *Denotes $p<0.05$ vs CON. Glucose time responses are presented as means only for clarity, whilst

AUCs are presented as mean change (bars) with individual change scores (dots) compared with CON

mean 24-h glucose can reflect changes during both 'ambient' and 'postprandial' periods (Monnier and Colette 2015). Nevertheless, it is reasonable to suggest that the $\sim 0.5 \mathrm{mmol} / \mathrm{l}$

average reduction in 24-h mean glucose and $\sim 2$-h reduction in the prevalence of postprandial hyperglycaemia (per day) observed with REHIT, when performed on a regular 
basis, would make a meaningful impact on overall glycaemia (i.e. $\mathrm{HbA}_{1 \mathrm{c}}$ ) and hence long-term risk (Monnier and Colette 2015). The lower glucose AUC (-11\%) observed following dinner further supports this assertion, given that the relative contribution of postprandial hyperglycaemia to overall glycaemic exposure is greater in patients with $\mathrm{HbA}_{1 \mathrm{c}} \leq 7.3 \%$ (Monnier and Colette 2015).

There is also a strong correlation between postprandial hyperglycaemia and the risk of adverse cardiovascular events (Coutinho et al. 1999). However, whether intervening to improve glycaemic control lowers cardiovascular risk over the long term is currently contentious (Wing et al. 2013). Nonetheless, combined with evidence that REHIT improves cardiorespiratory fitness and resting blood pressure with 8 weeks of thrice weekly training sessions (Ruffino et al. 2017), the current study provides further (tentative) evidence that REHIT favourably modifies the cardiovascular risk profile in those with type 2 diabetes. The lack of improvement in insulin sensitivity and glycaemic control reported 3 days after the final training session by Ruffino et al. (2017) suggests that the positive acute effects on glycaemic control are short lived. Future research should determine the optimal frequency of REHIT to maintain the acute benefits on glycaemic control.

The fact we could largely replicate the findings of previous studies on MICT and glycaemic control (van Dijk et al. 2012, 2013) validates our methodology and strengthens these preliminary findings with REHIT. However, it should be highlighted that the magnitude of the effects observed with both MICT and REHIT in the present study are smaller than in previous studies (van Dijk et al. 2012, 2013). We suspect that this is explained by the fact that our participants' type 2 diabetes was relatively well controlled according to $\mathrm{HbA}_{1 \mathrm{c}}$. Van Dijk et al. (2013) demonstrated that reductions in, for example, mean 24-h glucose were lower $(-0.6 \mathrm{mmol} / \mathrm{l} \mathrm{vs}-1.2 \mathrm{mmol} / \mathrm{l})$ in well controlled $\left(\mathrm{HbA}_{1 \mathrm{c}}<7.0 \%\right)$ compared with sub-optimally controlled $\left(\mathrm{HbA}_{1 \mathrm{c}}>7 \%\right)$ individuals with type 2 diabetes, respectively. The mean reductions in 24-h glucose of $\sim 0.6 \mathrm{mmol} / \mathrm{l}$ with REHIT and $\sim 0.4 \mathrm{mmol} / \mathrm{l}$ with MICT in the present study are, therefore, in line with the literature (Van Dijk et al. 2013).

The lack of statistically significant improvement in most glycaemic parameters with HIIT is an unexpected finding, particularly given the improvements observed with REHIT. Gillen et al. (2012) used a comparable HIIT protocol, trial design, and participants $(n=7)$ of similar diabetic status, and reported that HIIT markedly lowered average postmeal glucose spikes, as well as the glucose concentrations 60-120 min after the three post-exercise meals. In contrast, we observed no significant change in the AUC to any postexercise meal or in any other marker of postprandial glycaemia. Similar, however, was the lower prevalence of hyperglycaemia despite no significant change in mean 24-h glucose
(Gillen et al. 2012). It is important to highlight that the mean change for several of the glycaemic variables assessed with HIIT were in a favourable direction in the current study, but there was greater variation in individual change scores than with REHIT and MICT. It is possible that with an increased sample size and greater statistical power we would have observed effects of HIIT on other glycaemic parameters. Considering the accumulating evidence for beneficial training effects with this HIIT protocol in type 2 diabetes (Little et al. 2011; Francois et al. 2017), we would encourage larger definitive studies of the acute effects on glycaemic control.

There are several considerations in the study design and employed techniques that should be acknowledged. First, although we provided standardised food packages during all trials, and asked participants to record the time of their medication, the study was otherwise free-living and (although this is also a strength of the study) we only have participants' self-reported compliance. In addition, although CGM captures glycaemic data outside the laboratory and has shown good agreement with responses measured simultaneously in venous blood (Bailey et al. 2014), the coefficient of variation for some parameters can be high (Terada et al. 2014). The fact we could detect differences despite this lower level of control is encouraging, but we acknowledge that our small sample size, although consistent with numerous other studies on this topic (van Dijk et al. 2012, 2013; Manders et al. 2010), is a key limitation and our data should be interpreted with caution in that context. Larger free-living CGM studies combined with more controlled laboratory assessments will be required to confirm our preliminary findings. We were also only able to capture postprandial glucose responses in this study and can provide no mechanistic insight, and so future investigations should aim to provide a more holistic and mechanistic assessment of carbohydrate and lipid metabolism following REHIT in both men and women with type 2 diabetes.

In conclusion, this study suggests that a brief bout of REHIT improves markers of postprandial glycaemic control over the following 24-h period when compared with no exercise. We conclude that REHIT may offer a genuinely time-efficient exercise option for men with type 2 diabetes and warrants further study.

Acknowledgements All authors confirm that they have no conflict of interest to disclose. We would like to thank our participants for the considerable time and effort expended in completing this study, and our family and friends for their support during our academic endeavours. We would also like to acknowledge the assistance of N. McLaughlin and D. Fernie (Ulster University) during data collection. This work was supported by the Diabetes Research \& Wellness Foundation (Grant No. SCA/OF/12/15).

Author contributions RM conceived the study idea and designed the initial methodology. CM, BF, NB, SF and GD contributed to the refinement of the study methodology. RM, GM and SF collected the data 
with support from CM, BF, NB and GD. RM and GM analysed the data. RM wrote the initial draft of the manuscript. CM, GM, BF, NB, $\mathrm{SF}$ and GD critiqued the initial draft manuscript and contributed to the final submission. All authors approved the final submission.

Data availability The raw data for this study are available from the corresponding author on reasonable request.

Open Access This article is distributed under the terms of the Creative Commons Attribution 4.0 International License (http://creativeco mmons.org/licenses/by/4.0/), which permits unrestricted use, distribution, and reproduction in any medium, provided you give appropriate credit to the original author(s) and the source, provide a link to the Creative Commons license, and indicate if changes were made.

\section{References}

Allender S, Scarborough P, Peto V, Rayner M, Leal J, Luengo-Fernandez R, Gray A (2008) European cardiovascular disease statistics. European Heart Network, Brussels, England

Bailey TS, Ahmann A, Brazg R, Christiansen M, Garg S, Watkins E, Welsh JB, Lee SW (2014) Accuracy and acceptability of the 6-day Enlite continuous subcutaneous glucose sensor. Diabetes Technol Ther 16(5):277-283

Bantle JP, Wylie-Rosett J, Albright AL, Apovian CM, Clark NG, Franz MJ, Hoogwerf BJ, Lichtenstein AH, Mayer-Davis E, Mooradian AD, Wheeler ML, Association AD (2008) Nutrition recommendations and interventions for diabetes: a position statement of the American Diabetes Association. Diabetes Care 31(Suppl 1):S61-S78

Church TS, Blair SN, Cocreham S, Johannsen N, Johnson W, Kramer K, Mikus CR, Myers V, Nauta M, Rodarte RQ, Sparks L, Thompson A, Earnest CP (2010) Effects of aerobic and resistance training on hemoglobin A1c levels in patients with type 2 diabetes: a randomized controlled trial. JAMA 304(20):2253-2262

Colberg SR, Sigal RJ, Yardley JE, Riddell MC, Dunstan DW, Dempsey PC, Horton ES, Castorino K, Tate DF (2016) Physical activity/ exercise and diabetes: a position statement of the American Diabetes Association. Diabetes Care 39(11):2065-2079

Coutinho M, Gerstein HC, Wang Y, Yusuf S (1999) The relationship between glucose and incident cardiovascular events. A metaregression analysis of published data from 20 studies of 95,783 individuals followed for 12.4 years. Diabetes Care 22(2):233-240

Craig CL, Marshall AL, Sjostrom M, Bauman AE, Booth ML, Ainsworth BE, Pratt M, Ekelund U, Yngve A, Sallis JF, Oja P (2003) International physical activity questionnaire: 12-country reliability and validity. Med Sci Sports Exerc 35(8):1381-1395

Dela F, Larsen JJ, Mikines KJ, Ploug T, Petersen LN, Galbo H (1995) Insulin-stimulated muscle glucose clearance in patients with NIDDM. Effects of one-legged physical training. Diabetes 44(9):1010-1020

Francois ME, Durrer C, Pistawka KJ, Halperin FA, Chang C, Little JP (2017) Combined interval training and post-exercise nutrition in type 2 diabetes: a randomized control trial. Front Physiol 8:528

Garber CE, Blissmer B, Deschenes MR, Franklin BA, Lamonte MJ, Lee IM, Nieman DC, Swain DP, Medicine ACoS (2011) American College of Sports Medicine position stand. Quantity and quality of exercise for developing and maintaining cardiorespiratory, musculoskeletal, and neuromotor fitness in apparently healthy adults: guidance for prescribing exercise. Med Sci Sports Exerc 43(7):1334-1359
Gillen JB, Little JP, Punthakee Z, Tarnopolsky MA, Riddell MC, Gibala MJ (2012) Acute high-intensity interval exercise reduces the postprandial glucose response and prevalence of hyperglycaemia in patients with type 2 diabetes. Diabetes Obes Metab 14(6):575-577

Gillen JB, Martin BJ, MacInnis MJ, Skelly LE, Tarnopolsky MA, Gibala MJ (2016) Twelve weeks of sprint interval training improves indices of cardiometabolic health similar to traditional endurance training despite a five-fold lower exercise volume and time commitment. PLoS One 11(4):e0154075

Hallal PC, Andersen LB, Bull FC, Guthold R, Haskell W, Ekelund U, Group LPASW (2012) Global physical activity levels: surveillance progress, pitfalls, and prospects. Lancet 380(9838):247-57

Hardcastle SJ, Ray H, Beale L, Hagger MS (2014) Why sprint interval training is inappropriate for a largely sedentary population. Front Psychol 5:1505

Harris JA, Benedict FG (1918) A Biometric Study of Human Basal Metabolism. Proc Natl Acad Sci USA 4(12):370-373

Haskell WL, Lee IM, Pate RR, Powell KE, Blair SN, Franklin BA, Macera CA, Heath GW, Thompson PD, Bauman A (2007) Physical activity and public health: updated recommendation for adults from the American College of Sports Medicine and the American Heart Association. Med Sci Sports Exerc 39(8):1423-1434

Howley ET, Bassett DR, Welch HG (1995) Criteria for maximal oxygen uptake: review and commentary. Med Sci Sports Exerc 27(9):1292-1301

International Diabetes Federation Guideline Development Group (2014) Guideline for management of postmeal glucose in diabetes. Diabetes Res Clin Pract 103(2):256-268

Korkiakangas EE, Alahuhta MA, Laitinen JH (2009) Barriers to regular exercise among adults at high risk or diagnosed with type 2 diabetes: a systematic review. Health Promot Int 24(4):416-427

Little JP, Gillen JB, Percival ME, Safdar A, Tarnopolsky MA, Punthakee Z, Jung ME, Gibala MJ (2011) Low-volume high-intensity interval training reduces hyperglycemia and increases muscle mitochondrial capacity in patients with type 2 diabetes. J Appl Physiol 111(6):1554-1560

Little JP, Jung ME, Wright AE, Wright W, Manders RJ (2014) Effects of high-intensity interval exercise versus continuous moderateintensity exercise on postprandial glycemic control assessed by continuous glucose monitoring in obese adults. Appl Physiol Nutr Metab 39(7):835-841

Manders RJ, Van Dijk JW, van Loon LJ (2010) Low-intensity exercise reduces the prevalence of hyperglycemia in type 2 diabetes. Med Sci Sports Exerc 42(2):219-225

Maxwell SE, Delaney HD (2004) Designing experiments and analyzing data: a model comparison perspective, 2 ed. Lawrence Erlbaum Associates, London

Metcalfe RS, Babraj JA, Fawkner SG, Vollaard NB (2012) Towards the minimal amount of exercise for improving metabolic health: beneficial effects of reduced-exertion high-intensity interval training. Eur J Appl Physiol 112(7):2767-2775

Metcalfe RS, Koumanov F, Ruffino JS, Stokes KA, Holman GD, Thompson D, Vollaard NB (2015) Physiological and molecular responses to an acute bout of reduced-exertion high-intensity interval training (REHIT). Eur J Appl Physiol 115(11):2321-2334

Metcalfe RS, Tardif N, Thompson D, Vollaard NB (2016) Changes in aerobic capacity and glycaemic control in response to reducedexertion high-intensity interval training (REHIT) are not different between sedentary men and women. Appl Physiol Nutr Metab 41(11):1117-1123

Monnier L, Colette C (2015) Postprandial and basal hyperglycaemia in type 2 diabetes: contributions to overall glucose exposure and diabetic complications. Diabetes Metab 41(6 Suppl 1):6S9-6S15 
Morrato EH, Hill JO, Wyatt HR, Ghushchyan V, Sullivan PW (2007) Physical activity in US adults with diabetes and at risk for developing diabetes, 2003. Diabetes Care 30(2):203-209

Paneni F, Beckman JA, Creager MA, Cosentino F (2013) Diabetes and vascular disease: pathophysiology, clinical consequences, and medical therapy: part I. Eur Heart J 34(31):2436-2443

Pate RR, O'Neill JR, Lobelo F (2008) The evolving definition of "sedentary”. Exerc Sport Sci Rev 36(4):173-178

Rodbard D (2009) New and improved methods to characterize glycemic variability using continuous glucose monitoring. Diabetes Technol Ther 11(9):551-565

Ruffino JS, Songsorn P, Haggett M, Edmonds D, Robinson AM, Thompson D, Vollaard NB (2017) A comparison of the health benefits of reduced-exertion high-intensity interval training (REHIT) and moderate-intensity walking in type 2 diabetes patients. Appl Physiol Nutr Metab 42(2):202-208

Stolar M (2010) Glycemic control and complications in type 2 diabetes mellitus. Am J Med 123(3 Suppl):S3-S11

Terada T, Loehr S, Guigard E, McCargar LJ, Bell GJ, Senior P, Boulé NG (2014) Test-retest reliability of a continuous glucose monitoring system in individuals with type 2 diabetes. Diabetes Technol Ther 16(8):491-498

Terada T, Wilson BJ, Myette-Cóté E, Kuzik N, Bell GJ, McCargar LJ, Boulé NG (2016) Targeting specific interstitial glycemic parameters with high-intensity interval exercise and fasted-state exercise in type 2 diabetes. Metabolism 65(5):599-608

Thompson D, Batterham AM, Bock S, Robson C, Stokes K (2006) Assessment of low-to-moderate intensity physical activity thermogenesis in young adults using synchronized heart rate and accelerometry with branched-equation modeling. J Nutr 136(4):1037-1042

Townsend LK, Islam H, Dunn E, Eys M, Robertson-Wilson J, Hazell TJ (2017) Modified sprint interval training protocols. Part II. Psychological responses. Appl Physiol Nutr Metab 42(4):347-353 van Dijk JW, Manders RJ, Hartgens F, Stehouwer CD, Praet SF, van Loon LJ (2011) Postprandial hyperglycemia is highly prevalent throughout the day in type 2 diabetes patients. Diabetes Res Clin Pract 93(1):31-37

van Dijk JW, Manders RJ, Tummers K, Bonomi AG, Stehouwer CD, Hartgens F, van Loon LJ (2012) Both resistance- and endurancetype exercise reduce the prevalence of hyperglycaemia in individuals with impaired glucose tolerance and in insulin-treated and non-insulin-treated type 2 diabetic patients. Diabetologia 55(5):1273-1282

Van Dijk JW, Manders RJ, Canfora EE, Mechelen WV, Hartgens F, Stehouwer CD, Van Loon LJ (2013) Exercise and 24-h glycemic control: equal effects for all type 2 diabetes patients?. Med Sci Sports Exerc 45(4):628-635

van Dijk JW, Venema M, van Mechelen W, Stehouwer CD, Hartgens F, van Loon LJ (2013) Effect of moderate-intensity exercise versus activities of daily living on 24-hour blood glucose homeostasis in male patients with type 2 diabetes. Diabetes Care 36(11):3448-3453

Vollaard NB, Metcalfe RS (2017) Research into the health benefits of sprint interval training should focus on protocols with fewer and shorter sprints. Sports Med 47(12):2443-2451

Wing RR, Bolin P, Brancati FL, Bray GA, Clark JM, Coday M, Crow RS, Curtis JM, Egan CM, Espeland MA, Evans M, Foreyt JP, Ghazarian S, Gregg EW, Harrison B, Hazuda HP, Hill JO, Horton ES, Hubbard VS, Jakicic JM, Jeffery RW, Johnson KC, Kahn SE, Kitabchi AE, Knowler WC, Lewis CE, Maschak-Carey BJ, Montez MG, Murillo A, Nathan DM, Patricio J, Peters A, PiSunyer X, Pownall H, Reboussin D, Regensteiner JG, Rickman AD, Ryan DH, Safford M, Wadden TA, Wagenknecht LE, West DS, Williamson DF, Yanovski SZ, Group (2013) Cardiovascular effects of intensive lifestyle intervention in type 2 diabetes. N Engl J Med 369(2):145-154 